\title{
Little Brown Lemurs Come of Age: Summary and Perspective
}

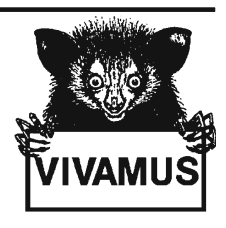

\author{
Ian Tattersall ${ }^{1}$ (D) Robert W. Sussman ${ }^{2}$
}

Received: 19 January 2016 / Accepted: 19 January 2016 / Published online: 13 February 2016

(C) Springer Science+Business Media New York 2016

The "little brown lemurs" (Tattersall and Sussman 1998) are all brownish or blackish arboreal quadrupeds of the genus Eulemur that closely resemble one another in body proportions, are small-bodied (in the full spectrum of lemur body sizes before the very recent anthropogenic extinctions), and are mainly frugivorous. Such characteristics make them analogous to the nondescript bird species that are often dismissed by ornithologists as "little brown jobs;" and it is only recently that they have attracted significant attention from primatologists. Still, this belated attention has paid major dividends, and it is gratifying how greatly our knowledge of this section of the genus Eulemur has expanded over the almost two decades since we first lamented the lack of scientific notice paid to its charming but unobtrusive constituents (Tattersall and Sussman 1998). As the contributions to this Special Issue exemplify, ecologists, behaviorists, conservationists, and others have showered unprecedented attention on members of the group, so that we now have a much wider and more balanced appreciation of the behavioral and ecological ranges they display. What is more, observations on the brown lemurs have acted as the springboard for investigation of a variety of more general questions in behavior and physiology. At the same time, however, arbitrary systematic revision of the group has created uncertainties that are hardly helpful to these burgeoning studies.

Back in 1998, consensus still held that all of the brown lemurs belonged to the single species Eulemur fulvus, one of five within its genus. Eulemur fulvus itself was highly polytypic, containing six distinctive subspecies that were clearly differentiated by pelage coloration and were mostly sexually dichromatic as well. All were essentially

Ian Tattersall

iant@amnh.org

Robert W. Sussman

rwsussma@artsci.wustl.edu

1 American Museum of Natural History, New York, NY 10024, USA

2 Department of Anthropology, Washington University, St. Louis, MO 63130, USA 
allopatric, although outside the accepted distributions a few field observers had also noted some brown lemur variants that did not fit easily into any of the established categories. As a whole, Eulemur fulvus appeared as a monophyletic clade of distinctive but reproductively compatible subspecies (except, apparently, in the case of Eulemur collaris/albocollaris), the distributions of which were geographically superimposed upon the earlier radiation that embraced the five recognized species of Eulemur, including E. fulvus itself.

All this began to change dramatically at the beginning of this millennium, with the publication of Colin Groves' Primate Taxonomy (2001) and, more influentially, with the second edition of the Lemurs of Madagascar field guide (Mittermeier et al. 2006). In both of these works, each of the former subspecies of Eulemur fulvus (fulvus, rufus, collaris, albocollaris, albifrons, and sanfordi) was raised to full species status, fully equivalent to E. macaco (by now itself polytypic), mongoz, coronatus, and rubriventer. In the third edition of the field guide (Mittermeier et al. 2010) things became yet more complicated, with the resurrection (following a suggestion initially made by Groves) of the name Eulemur cinereiceps for the form earlier termed E. albocollaris, and the splitting of the rufus population into E. rufifrons in the south and E. rufus in the north. In addition, both subspecies of black lemur were raised to species level, giving E. macaco and E. flavifrons.

Thus, from containing a mere five species in 1998, one of them polytypic, the genus Eulemur has now swelled to 12 species, none of them polytypic. The main justification for this expansion appears to have been Groves' adoption of a fundamentalist version of Joel Cracraft's Phylogenetic Species Concept (Cracraft 1989) that procedurally reduces species to irreducible diagnosable units. This approach has done little to illuminate what has evidently been a very intricate biological history. Both morphological (Tattersall and Schwartz 1991) and genomic (Markolf et al. 2013) analyses make it clear that the brown lemurs constitute a monophyletic unit of more recent origin than the broader Eulemur radiation from which they emerged. All of this subgroup's components (except possibly for cinereiceps: see Johnson et al. this issue) appear functionally interfertile, while morphological and genomic variation within it sorts along well defined geographic lines.

Clearly, what we see today among the brown lemurs reflects a complex and dynamic history of differentiation, doubtless played out against an equally eventful climatic and environmental background. We need to recognize that the intricacies of this process are likely to be lost if we insist on the reductionist pigeonholing of irreducible players in this rich evolutionary game as if they were fixed chess pieces. Much more was going on here than this static vision portends. The complex differentiation of the brown lemur group still offers us plenty of biological and nomenclatural questions that require resolution; and doing so needs to be a priority, given that these issues potentially impact comparative analyses such as those offered in this issue by Gamba et al. Donati et al., Sato et al., Gordon et al., and Kamilar and Tecot.

The unusual activity pattern that has come to be known as cathemerality (Tattersall 1988) was first identified in a population of brown lemurs on the island of Mayotte, in the Comoro group (Tattersall 1979). The rhythms of most primates are fairly straightforwardly governed by the day/night cycle; but among those Comorian Eulemur fulvus, living in secondary lowland forests, activity was unexpectedly partitioned fairly equally between day and night, year-round. Meanwhile, surveys on the neighboring Anjouan 
Island had suggested that Eulemur mongoz were active diurnally in the cooler highlands during the transition between the dry and rainy seasons, while in the warmer lowlands they were nocturnal at the same time of year (Tattersall 1976). How this pattern may or may not have related to the kind of cathemerality observed in Mayotte was unknown. As a result, when we first wrote in 1998 about the need for greater scrutiny of the little brown lemurs, we were unable to do more than suggest that cathemerality - as very broadly defined by quasi-equal distribution of activity between day and night - might also characterize E. macaco and other members of the Eulemur group. At that point, too little was known to allow us to begin sketching a larger picture.

Happily, the situation has since changed radically. And, as one might have expected, the issue has turned out to be much more complex than initially suspected. There is now an extensive literature demonstrating at least occasional cathemeral activity in all Eulemur species, as well as in Hapalemur, Varecia, and Lemur, and even in a couple of South American monkeys (Fernandez-Duque and Erkert 2006). Indeed, as far back as a decade ago sufficient detailed observations had already been gathered for Curtis and Rasmussen (2006) to recognize three distinct modes of cathemerality, governed by seasonality profiles. Other authors have since begun to investigate both the physiological mechanisms underlying cathemerality and the impacts on its expression of varying environmental stimuli. It has even been suggested that cathemerality was ancestral not only for the genus Eulemur, but also for Lemuridae in general (Curtis and Rasmussen 2006; Santini et al. 2015). As a result, the recognition of this unheralded pattern among the little brown lemurs has spurred investigation not only of cathemerality itself, but also of how primates more generally respond to a whole range of environmental stimuli in their activity distributions.

In an analysis reported in this volume, Donati et al. review long-term studies of 13 species of Eulemur conducted since 1989, with the aim of examining the conditions that potentially lead to cathemeral activity. They conclude that the higher the proportion of leaves in the diet of brown lemurs, the more similar the annual proportions of diurnal and nocturnal activity. However, they also find that Eulemur species are more nocturnal at sites with higher levels of human disturbance such as hunting, logging, and fragmentation. This latter observation is supported by earlier data on populations of E. collaris living in more and less disturbed habitats in southeastern Madagascar. Donati and colleagues also find that group size is a strong positive predictor of the level of diurnality among the 13 populations of Eulemur. Those that are pair-bonded (such as E. rubriventer and E. mongoz) are more nocturnal than other species, just as smaller groups of E. collaris living in southeastern Madagascar are likewise more inclined to nocturnality. As predicted, Eulemur tends to be more diurnal in areas of low overall human disturbance, while greater nocturnality is associated with higher human hunting pressures. Indirect effects that also promote nocturnality include habitat degradation, increased exposure to predators, lack of food resources, and climatic extremes.

The little brown lemurs have turned out to be remarkably varied in social behavior and organization, to have very diverse and adaptable diets, and to exhibit very flexible behaviors and ecological preferences. Their vocalizations are generally similar, although a variety of specific calls has been identified. Gamba et al. report (this issue) that, among fulvus, coronatus, flavifrons, rufus, macaco, mongoz, and rubriventer, call repertoire size varies from 9 to 14, with a mean of 11 . Although most eulemurs live in 
multimale-multifemale groups, E. rubriventer and E. mongoz are pair-bonded. As discussed by Kappeler and Fichtel (this issue), this form of social organization seems to have evolved from a group-living ancestral condition. Kappeler and Fichtel do not believe that the transition to pair-bonding was driven by male infanticide, social instability, or any single ecological factor. They propose instead that female competition favored small female group size, and that competition with sympatric congeners exacerbated the evolution of pair-bonding. In addition, one might also suggest that such factors as a largely nocturnal activity cycle (see preceding text), and specific choices of foods (such as a local dependence on flowers) might be examined as possible promotors of pair formation.

We have already noted that, regardless of whatever taxonomy is preferred, the group containing the lemur populations formerly regarded as subspecies of Eulemur fulvus appears monophyletic. Geographic distributions within it more or less map on to those of E. mongoz, E. macaco, E. coronatus, and E. rubriventer as one moves southwards on the north, northwest, and eastern coasts of Madagascar. Although there is some overlap, most sympatry and interspecies interaction within Eulemur occurs between members of the E. fulvus group and the latter four species. Data presented in this volume suggest that Eulemur hybrid zones represent stable, long-term interactions among parental species; and indeed, the ecological patterns underlying sympatry in Eulemur populations do not appear to differ greatly from what researchers have found among other primate genera. For example, as reported by Johnson and colleagues (this issue), Eulemur rufifrons and E. cinereiceps hybridize in the Andringitra region of southeastern Madagascar. The shapes and noncoincidence of genetic and morphological clines across the zone suggest there is no strong selection against hybrid forms (Delmore et al. 2011, 2013). Hybrids appear to diverge in ecological adaptation such as overall and scarce-season diet (Johnson 2007), and both visual inspection and identity tests indicate divergence, i.e., possession of nonidentical niches, among the hybrid and parental populations, which seem to be in a stable equilibrium. Hybrids are particular to specific sites, where they are apparently as fit as the parental forms, occupying a novel habitat that is characterized both by greater seasonality in precipitation and lower seasonality in temperature (Delmore et al. 2013). Furthermore, there are significant differences in the feeding ecologies of the hybrid and parental forms, including different diets during seasons with low food abundance (Johnson 2002, 2007). Johnson et al. (this issue) thus conclude that all three have distinct niches and that the hybrids exist relatively independent of both parental forms, perhaps exhibiting the early stages of speciation. This of course has potential relevance to the issues of species-level systematics within the group discussed in the preceding text.

The extreme adaptability and variability of the Eulemur fulvus group makes this monophyletic assemblage hugely interesting ecologically. The diverse relationship of cathemeral activity to climatic, ecological, and anthropogenic factors confirms that both habitat and level of protection affect activity cycles at specific sites. Eulemur eat not only the fruit but also the leaves of many native and introduced plant species, and often rely on variable, patchy resources (Sato et al. this issue). However, both Sato et al. and Sussman (1977) found that the dietary flexibility of Propithecus was actually greater, with Eulemur species being relatively highly frugivorous with lesser levels of dietary flexibility. Sato and colleagues further find that, when fruits are available, eulemurs adopt a "power-feeding" strategy (consuming large volumes of food per unit time) by 
increasing their daily path length, expanding their ranging areas, and adopting fissionfusion social organization. Eulemurs tend also to adopt a cathemeral feeding schedule when feeding on low-quality foods. Thus, these lemurs appear to change feeding strategies quite readily in response to the varying availability of fruit resources, exhibiting a behavioral flexibility that allows them to compensate for their lack of specialized physio-anatomical adaptations. These opportunistic responses to resource availability may deeply affect the seed shadow patterns of plant species dispersed by Eulemur, with brown lemurs facilitating recovery of native vegetation by transporting seeds from mature to disturbed forest patches. Most remarkable of all, perhaps, is the ability of brown lemurs to display cathemeral activity under a number of ecological conditions. This plasticity may explain why Eulemur fulvus is an important constituent of a wider range of habitats than any other genus of "diurnal" lemur on the island, something that may allow populations of Eulemur to persist in disturbed areas longer than other lemurs with less flexible activity patterns (see Donati et al. this issue).

Gordon and colleagues (this issue) attempt to determine whether body size among eulemurs correlates with ecological parameters such as overall food availability or resource seasonality, or alternatively with temperature, i.e., Bergmann's rule. They studied 11 of the 12 described Eulemur species, finding that body size variation across Eulemur is generally consistent with Bergmann's rule: about $40 \%$ of variation in body size across populations is accounted for by variation in ambient temperature and body mass is highest in areas that experience the coldest minimum temperatures. In contrast, variations in body mass do not mirror resource seasonality as they do in other lemur taxa such as Propithecus. The authors suggest that future comparative studies should attempt to determine whether the presence or absence of patterns observed at high taxonomic levels is consistent with what is observed at lower taxonomic levels within the same data sets. If not, there should be attempts to determine whether additional factors might be involved in these relationships, e.g., dietary niche, activity pattern, and life history variables.

Overall, given the current behavioral range of the genus, Eulemur seems more ecologically resilient to current threats than the larger-bodied, more specialized sifakas. However, Eulemur species are not immune to the negative impacts of habitat disturbance. The forests of Madagascar are being devastated at a remarkable rate, and no species of lemur lives without the danger of vast reduction and depletion (Green and Sussman 1990; Schwitzer et al. 2014). For example, Eulemur albocollaris lives in areas of widespread habitat destruction, experiences heavy hunting pressures, and has low population densities. It is among the most endangered primate species in Madagascar (Johnson 2007). In the most southwestern portion of its range Eulemur rufifrons is also being reduced at an alarming rate due to human habitat destruction (Kelley et al. 2007).

Using a species distribution modeling approach, Kamilar and Tecot (this issue) find that adding anthropogenic factors substantially improves the predictive value of climate-based models of Eulemur distributions. In particular, five Eulemur species (E. collaris, E. fulvus, E. flavifrons, E. rufifrons, and E. sanfordi) exhibit substantially reduced distributions once anthropogenic effects are accounted for. In most cases, the distance to dense settlements is the most significant negative anthropogenic influence, followed by distance to cropland, and then by distance to smaller villages. Factors correlating with lower lemur populations in affected areas include higher fecal glucocorticoid metabolite levels, difficulties in responding behaviorally or physiologically to 
seasonal changes in food availability and climate, higher infant mortality rates, and pathogen transmission between humans and lemurs, as well as higher rates of hunting of the animals. To these, we would add high and increasing rates of destruction of primary forest.

Kamilar and Tecot's review reminds us what a tremendously negative influence human impacts have on primate distributions, and how much those impacts increase local extinction risk. Fortunately, as so many of the contributions to this Special Issue emphasize, there is little doubt that the little brown lemurs of Madagascar are remarkably adaptable on the behavioral level; and, as hardy survivors, they are indeed worthy of our admiration. Sadly, though, the underlying subtext of many of the articles presented in this issue is that adaptability has its limits.

Acknowledgments We thank Giuseppe Donati and Steig Johnson for their kind invitation to contribute to this Special Issue on Eulemur, a genus dear to our hearts.

\section{References}

Cracraft, J. (1989). Speciation and its ontology. In D. Otte \& J. Endler (Eds.), Speciation and its consequences (pp. 28-59). Sunderland, MA: Sinauer.

Curtis, D. J., \& Rasmussen, M. A. (2006). The evolution of cathemerality in primates and other mammals: a comparative and chronoecological approach. Folia Primatologica, 77(1-2), 178-193.

Delmore, K. E., Louis, E. E., \& Johnson, S. E. (2011). Morphological characterization of a brown lemur hybrid zone (Eulemur rufifrons $\times$ E. cinereiceps). American Journal of Physical Anthropology, 145, 5566.

Delmore, K. E., Brenneman, R. A., Lei, R., Bailey, C. A., Brelsford, A., Louis, E. E., \& Johnson, S. E. (2013). Clinal variation in a brown lemur (Eulemur spp.) hybrid zone: combining morphological, genetic and climatic data to examine stability. Journal of Evolutionary Biology, 26, 1677-1690.

Fernandez-Duque, E., \& Erkert, H. G. (2006). Cathemerality and lunar periodicity of activity rhythms in owl monkeys of the Argentinian Chaco. Folia Primatologica, 77, 123-138.

Green, G. M., \& Sussman, R. W. (1990). Deforestation history of the eastern rain forests of Madagascar from satellite images. Science, 248(4952), 212-215.

Johnson, S. E. (2002). Ecology and speciation in brown lemurs: White-collared lemurs (Eulemur albocollaris) and hybrids (Eulemur albocollaris $\times$ Eulemur fulvus rufus) in southeastern Madagascar. Ph.D. dissertation, University of Texas at Austin.

Johnson, S. E. (2007). Evolutionary divergence in the brown lemur species complex. In L. Gould \& M. L. Sauther (Eds.), Lemurs: Ecology and adaptation (pp. 187-210). New York: Springer Science+Business Media.

Kelley, E. A., Sussman, R. W., \& Muldoon, K. M. (2007). The status of lemur species at Antserananomby: an update. Primate Conservation, 22, 71-77.

Markolf, M., Rakotonirina, H., Fichtel, C., von Grumbkow, P., Brameier, M., \& Kappeler, P. M. (2013). True lemurs...true species: species delimitation using multiple data sources in the brown lemur complex. $B M C$ Evolutionary Biology, 13, 233.

Mittermeier, R. A., Konstant, W. R., Hawkins, F., Louis, E. E., Langrand, O., Ratsimbazafy, J., Rasoloarison, R., Ganzhorn, J. U., Rajaobelina, S., Tattersall, I., \& Meyers, D. M. (2006). Lemurs of Madagascar (2nd ed.). Washington, DC: Conservation International.

Mittermeier, R. A., Louis, E. E., Richardson, M., Schwitzer, C., Langrand, O., Rylands, A. B., Hawkins, F., Rajaobelina, S., Ratsimbazafy, J., Rasoloarison, R., Roos, C., Kappeler, P. M., \& MacKinnon, J. (2010). Lemurs of Madagascar (3rd ed.). Washington, DC: Conservation International.

Santini, L., Rojas, D., \& Donati, G. (2015). Evolving through day and night: origin and diversification of activity pattern in modern primates. Behavioural Ecology, 26(3), 789-796.

Schwitzer, C., Mittermeier, R. A., Johnson, S. E., Donati, G., Irwin, M., Peacock, H., Ratsimbazafy, J., Razafindramanana, J., Louis, E. E., Jr., Chikhi, L., Colquhoun, I. C., Tinsman, J., Dolch, R., LaFleur, M., 
Nash, S., Patel, E., Randrianambinina, B., Rasolofoharivelo, T., \& Wright, P. C. (2014). Averting lemur extinctions amid Madagascar's political crisis. Science, 343(6173), 842-843.

Sussman, R. W. (1977). Feeding behavior in Lemur catta and Lemur fulvus. In T. Clutton-Brock (Ed.), Primate ecology: Feeding and ranging behavior in lemurs, monkeys, and apes (pp. 1-36). London: Academic Press.

Tattersall, I. (1976). Group structure and activity rhythm in Lemur mongoz (Primates, Lemuriformes) on Anjouan and Moheli islands, Comoro archipelago. Anthropological Papers of the American Museum of Natural History, 53(4), 363-378.

Tattersall, I. (1979). Patterns of activity in the Mayotte lemur, Lemur fulvus mayottensis. Journal of Mammalogy, 60(2), 314-323.

Tattersall, I. (1988). Cathemeral activity in primates: a definition. Folia Primatologica, 49, 200-202.

Tattersall, I., \& Schwartz, J. H. (1991). Phylogeny and nomenclature in the Lemur-group of Malagasy strepsirhine primates. Anthropological Papers of the American Museum of Natural History, 69, 1-18.

Tattersall, I., \& Sussman, R. (1998). "Little brown lemurs" of northern Madagascar. Folia Primatologica, 69(Suppl. 1), 379-388. 